\title{
Prevalencia de tabaquismo durante el embarazo en mujeres chilenas de bajo nivel socioeconómico
}

\author{
JAVIER MALLOL V.*, DIEGO BRANDENBURG J.*, RICARDO MADRID H.*, \\ FERNANDO SEMPERTEGUI G.*, LUIS RAMÍREZ A.** y DANIEL JORQUERA A.**
}

\author{
Prevalence of tobacco smoking during pregnancy in Chilean \\ women of low socioeconomic status
}

Tobacco smoking has increased among young women in reproductive age from developing countries but its prevalence during pregnancy is virtually unknown. This study describes the prevalence of smoking during pregnancy in a random sample of 400 mothers of low socioeconomic status from South-Santiago, Chile. The prevalence of active tobacco smoking during pregnancy was $28 \%$. Fifty-nine percent of mothers who had never smoked were exposed to intra-domiciliary tobacco smoke during pregnancy. There was no significant difference in birth weight, height, and Apgar score between exposed and non-exposed groups. However, the newborns from mothers who were active smokers previous to pregnancy presented lower birth weight $(p=0.04)$ and higher frequency of low weight for gestacional age $(p=0.02)$ than newborns from non-smoking mothers. The prevalence of tobacco smoking during pregnancy in mothers of low socioeconomic status is as higher as that reported from developed countries. A high proportion of mothers (smokers and nonsmokers) are passively exposed to tobacco smoke at home during pregnancy.

Key words: Smoking, pregnancy, gestational smoking prevalence.

\section{Resumen}

El tabaquismo en mujeres jóvenes en edad fértil ha aumentado en los últimos años en países en desarrollo, pero su prevalencia en mujeres embarazadas de bajos recursos económicos es virtualmente desconocida. Este estudio describe la prevalencia del tabaquismo durante el embarazo en una muestra aleatoria de 400 madres de bajo nivel socioeconómico, atendidas en el Hospital El Pino, Santiago Sur, Chile. La prevalencia de tabaquismo durante el embarazo fue $28 \%$. Un alto porcentaje (59\%) de madres embarazadas que nunca habian fumado, estuvieron expuestas al humo del tabaco en sus hogares. No hubo una diferencia significativa entre los dos grupos en el peso, talla, puntaje de Apgar, o edad gestacional. Los recién nacidos (RN) de madres que eran fumadoras antes del embarazo presentaron menor peso de nacimiento que los $R N$ de madres no fumadoras $(p=0,04)$ $y$ mayor frecuencia de $R N$ pequeños para edad gestacional $(p=0,02)$. La prevalencia de tabaquismo durante el embarazo en madres de bajo nivel socioeconómico es alta y similar a las encontradas en mujeres embarazadas de países desarrollados. Una alta proporción de las madres embarazadas tanto fumadoras como no fumadoras está expuesta al humo de tabaco en sus hogares.

Palabras clave: Tabaco, embarazo, prevalencia de tabaquismo.

\footnotetext{
* Neumólogos Pediatras, Departamento de Medicina Respiratoria Infantil, Hospital El Pino, Universidad de Santiago de Chile (USACH).

** Internos de $7^{\circ}$ año de Medicina, Universidad de Santiago de Chile (USACH). Departamento de Medicina Respiratoria Infantil, Universidad de Santiago de Chile, Hospital CRS El Pino, Santiago, Chile.
} 


\section{Introducción}

El tabaquismo constituye la principal causa de muerte evitable en el mundo y en nuestro país sigue siendo uno de los principales problemas de salud pública. Estaría asociado a más de 4 millones de muertes anuales en todo el mundo y de casi 14.000 en Chile. La OMS ha informado que la prevalencia anual promedio de tabaquismo en el mundo es de aproximadamente $30 \%$, con amplias variaciones territoriales, culturales y económicas ${ }^{1}$. Según información reciente del Consejo Nacional para el Control de Estupefacientes (CONACE) ${ }^{2}$, organismo dependiente del Ministerio del Interior de Chile, en nuestro país la cifra de prevalencia anual de consumo de tabaco en la última década (19942004) ha permanecido estable en hombres (de $43,3 \%$ a $44,8 \%$ ) y ha presentado un significativo aumento entre las mujeres (de $35 \%$ a $40 \%$ ) en el mismo período. Sin embargo, se ha producido un alarmante incremento en la prevalencia de tabaquismo en mujeres entre los 19 y 25 años; desde un $42,5 \%$ en 1994 hasta un $55 \%$ en 2004. Este aumento cobra mucho mayor importancia si se considera que muchas de las mujeres fumadoras en edad reproductiva continúan fumando durante el embarazo, en especial las más jóvenes ${ }^{3}$.

Aunque los efectos deletéreos de la exposición al humo de tabaco durante el embarazo, tanto en la salud de la madre como del hijo, sean ampliamente conocidos, existe muy poca información acerca de la prevalencia de tabaquismo durante el embarazo, en particular en poblaciones de bajo nivel socioeconómico. El objetivo de este trabajo descriptivo fue determinar la prevalencia de tabaquismo durante el embarazo, y otras características del consumo de tabaco, en mujeres que se atienden en la maternidad del Hospital El Pino, situado en el área sur de Santiago, Chile.

\section{Material y Método}

La investigación se llevó a cabo en el Hospital CRS El Pino, el cual atiende a pacientes provenientes del sector sur de Santiago (comunas de San Bernardo y El Bosque), Chile. En esta maternidad se atienden en promedio 3.800 partos anuales y sirve a una población de bajos recursos socioeconómicos (Encuesta Casen, MIDEPLAN, Chile; www.mideplan.cl) provenientes de las comunas mencionadas. Cuatro- cientas de estas madres fueron aleatoriamente seleccionadas para responder un cuestionario con preguntas relativas al tabaquismo activo (uno o más cigarrillos diarios) y pasivo (inhalación involuntaria de humo de tabaco por presencia de fumadores en su casa), dirigido en especial a tabaquismo durante el embarazo. Todas ellas fueron entrevistadas por un médico pediatra durante su hospitalización en los primeros días del puerperio.

El cuestionario incluía preguntas sobre el consumo de tabaco de las madres tales como: tabaquismo diario previo al embarazo (fumaba todos los días desde que empezó a fumar) y ocasional (fumaba "a veces"), tabaquismo en el puerperio inmediato (presencia de consumo de tabaco en el momento de la aplicación de la encuesta), tabaquismo durante el embarazo (1 ó más cigarrillos diarios), período de la gestación en que fumó $\left(1^{\circ}, 2^{\circ}\right.$ ó $3^{\circ}$ trimestre), edad de inicio del tabaquismo, suspensión del consumo de tabaco debido al embarazo, exposición intradomiciliaria al humo de tabaco durante el embarazo y comorbilidades durante la gestación (anemia, colestasia, infección urinaria, toxemia, amenaza de aborto, amenaza de parto prematuro, diabetes gestacional, retardo del crecimiento intrauterino, u otros).

Además se registraron datos respecto a peso, talla, Apgar al primer y quinto minuto, y edad gestacional de los recién nacidos $(\mathrm{RN})$. El peso de los RN fue obtenido en una balanza electrónica (Seca ${ }^{\mathrm{TM}}$ modelo 727), y se consideró como RN de bajo peso los que pesaban menos de $2.500 \mathrm{~g}$. La clasificación de la edad gestacional como adecuados, grandes o pequeños se basó en la curva de crecimiento intrauterino de Juez et $\mathrm{al}^{4}$. Por su parte se definió como prematuros a los menores de 37 semanas de edad gestacional.

Los datos obtenidos fueron analizados empleando un programa estadístico (SPSS), utilizando estadísticos descriptivos, pruebas paramétricas para variables continuas de distribución normal, tablas de contingencia, ANOVA y $\chi^{2}$ para comparación de proporciones y tendencia lineal, según correspondiera. Se consideró estadísticamente significativo un valor de $\mathrm{p}<0,05$. Los resultados se expresan como promedio e intervalos de confianza (IC) del 95\%. El número requerido de embarazadas para un intervalo de confianza de $95 \%$ entre $23,5 \%$ y $32,5 \%$ con un $28 \%$ de prevalencia, es de 383 .

El presente estudio fue aprobado por el comité de ética del Hospital El Pino y todas las madres firmaron el consentimiento plenamente informado previo a su participación. 


\section{Resultados}

De las 400 madres seleccionadas, 397 fueron satisfactoriamente encuestadas para este estudio. La prevalencia total de tabaquismo previo al embarazo fue de $68 \%$, con un $45,1 \%$ de fumadoras diarias y $22,9 \%$ fumadoras ocasionales. Las madres fumadoras informaron que el $95,4 \%$ fumaba entre 1 y 10 cigarrillos/día; el 3,2\% fumaba entre 11 y 20 y el 1,4\% fumaba más de 20 cigarrillos/día. Con respecto a las mujeres que fumaban antes del embarazo y que también fumaron durante éste, el $50,3 \%$ era fumadoras diarias y sólo el 9,6\% eran fumadoras ocasionales. El 43,3\% de las madres fumadoras que dijeron haber dejado de fumar cuando supieron que estaban embarazadas, fumaron posteriormente en el transcurso del embarazo, sin que hubiera concordancia entre dejar de fumar por embarazo y no fumar durante el embarazo (kappa $0,017, p=0,79$ ).

La prevalencia total de tabaquismo durante el embarazo fue de $28 \%$. En relación a la prevalencia por trimestre, se observó una disminución significativa en la prevalencia de tabaquismo durante el embarazo con un $27 \%$ en el primer trimestre, $14,6 \%$ en el segundo y $12,3 \%$ en el tercero. La prevalencia de tabaquismo durante el embarazo entre las madres adolescentes fue significativamente mayor que entre las madres mayores de 19 años (46,7\% versus $26,7 \%$ ); hubo también una proporción significativamente mayor de madres adolescentes entre las madres que fumaron durante el embarazo en relación con las madres no fumadoras $(18,9 \%$ versus $8,4 \%$ ). Sin embargo, no hubo diferencia signifi- cativa entre la edad promedio de las madres fumadoras $(24,3$ años, IC95\% 23,1-25,6) y no fumadoras (25,3 años, IC95\% 24,5-26,1) durante el embarazo.

Las madres fumadoras durante el embarazo también estuvieron significativamente más expuestas al humo de tabaco intradomiciliario en relación a las no fumadoras $(79,3 \%$ versus $65,7 \%$ ), con una prevalencia total de tabaquismo pasivo intradomiciliario de $68 \%$ para el total de la muestra seleccionada. Se destaca el alto porcentaje de madres no fumadoras $(59 \%)$ que estuvieron expuestas al humo de tabaco intradomiciliario durante su embarazo. El porcentaje de madres que ya había fumado uno o más cigarrillos por día en los primeros días después del parto fue de un $12,6 \%$ y todas fueron madres que fumaron durante el embarazo. Las características del consumo de tabaco materno durante el embarazo se describen en la Tabla 1.

Con respecto a los RN (niñas: 49,9\%), no hubo diferencia significativa en el Apgar del primer y quinto minuto, peso, talla, edad gestacional, prematurez o bajo peso de nacimiento, de acuerdo a si fumaron o no durante el embarazo, aunque el grupo de niños cuyas madres fumaron durante el embarazo tuvo un peso promedio inferior (3.336 g, IC95\% 3.277-3.396) a aquel cuyas madres no fumaron $(3.424 \mathrm{~g}$, IC95\% 3.341-3.508), $\mathrm{p}=0,08$.

Cuando se estratificó la muestra por la variable fumadora diaria u ocasional previo al embarazo, el peso promedio de los $\mathrm{RN}$ de madres fumadoras diarias $(3.269 \mathrm{~g}$, IC95\% 3.195-3.341) fue significativamente menor que los $\mathrm{RN}$ de madres fumadoras ocasionales $(3.447 \mathrm{~g}$, IC95\%

Tabla 1. Características del consumo de tabaco en madres fumadoras y no fumadoras durante el embarazo

\begin{tabular}{|c|c|c|c|c|c|}
\hline & & $\begin{array}{c}\text { Madre fumó } \\
\text { durante embarazo } \\
(\mathrm{n}=111) \\
\%\end{array}$ & $\begin{array}{c}\text { Madre no fumó } \\
\text { durante embarazo } \\
(\mathrm{n}=286) \\
\%\end{array}$ & $\begin{array}{c}\text { Total } \\
\left(\begin{array}{c}(\mathrm{n}=397) \\
\%\end{array}\right.\end{array}$ & $\mathbf{p}$ \\
\hline \multirow[t]{4}{*}{ Edad } & $<18$ años & 18,9 & 8,4 & 11,3 & $<0,01$ \\
\hline & 18-25 años & 41,4 & 48,3 & 46,3 & 0,22 \\
\hline & 26-35 años & 31,5 & 33,9 & 33,2 & 0,65 \\
\hline & $>36$ años & 8,1 & 9,4 & 9,1 & 0,68 \\
\hline \multicolumn{2}{|c|}{ Tabaquismo previo al embarazo } & 97,3 & 40,2 & 68,0 & $<0,01$ \\
\hline \multicolumn{2}{|c|}{ Tabaquismo en primeros días del puerperio } & 45,0 & 0 & 12,6 & $<0,01$ \\
\hline \multicolumn{2}{|c|}{ Seguirá fumando } & 15,3 & 0,3 & 4,5 & $<0,01$ \\
\hline \multicolumn{2}{|c|}{ Tabaquismo intradomiciliario } & 79,3 & 60,5 & 65,7 & $<0,01$ \\
\hline \multicolumn{2}{|c|}{ Suspendió tabaco por embarazo } & 46,8 & 23,8 & 30,2 & $<0,01$ \\
\hline \multicolumn{2}{|c|}{ Otras patologías del embarazo } & 33,3 & 35,7 & 35,0 & 0,66 \\
\hline
\end{tabular}


Tabla 2. Características de los recién nacidos de acuerdo a si sus madres fumaban diariamente u ocasionalmente antes del embarazo

\begin{tabular}{|c|c|c|c|c|}
\hline & $\begin{array}{l}\text { Fumadoras diarias } \\
\qquad(\mathrm{n}=179)\end{array}$ & $\begin{array}{l}\text { Fumadoras ocasionales } \\
\qquad(\mathrm{n}=91)\end{array}$ & $\begin{array}{c}\text { Total } \\
(\mathrm{n}=397)\end{array}$ & $\mathbf{p}$ \\
\hline Apgar $1^{\circ}$ min.* & $8,3 \quad(8,1-8,5)$ & $8,1 \quad(7,7-8,6)$ & $8,3 \quad(8,1-8,4)$ & 0,43 \\
\hline Apgar $5^{\circ}$ min.* & $(8,9-9,0)$ & $8,9 \quad(8,9-9,0)$ & $9,0 \quad(8,9-9,0)$ & 0,14 \\
\hline Peso al nacer $(\mathrm{g})^{*}$ & $(3.195-3.341)$ & $3.447 \quad(3.330-3.562)$ & $(3.316-3.413)$ & 0,04 \\
\hline Talla al nacer $(\mathrm{cm})^{*}$ & $(49,7-50,3)$ & $50,4 \quad(50,0-50,8)$ & $(50,0-50,4)$ & 0,60 \\
\hline Edad gestacional (sem)* & $(38,6-39,1)$ & $38,8 \quad(38,5-39,0)$ & $(38,6-39,0)$ & 0,46 \\
\hline Prematuros (\%) & 6,7 & 7,8 & 7,3 & 0,70 \\
\hline Bajo peso nacimiento (\%) & 4,5 & 2,3 & 3,3 & 0,27 \\
\hline PEG (\%) & 17,9 & 9,6 & 13,4 & 0,02 \\
\hline AEG $(\%)$ & 74,9 & 78,6 & 76,8 & 0,41 \\
\hline GEG (\%) & 7,3 & 11,9 & 9,8 & 0,13 \\
\hline
\end{tabular}

* Resultados expresados en promedio (IC95\%). PEG, AEG y GEG = Pequeño, Adecuado y Grande para edad gestacional, respectivamente.

3.330-3.562), con una diferencia promedio de $178 \mathrm{~g}$ y $\operatorname{los} \mathrm{RN}$ de madres no fumadoras $(3.405$ g, IC95\% 3.330-3.480), $\mathrm{p}=0,04$. Además, se observó un porcentaje significativamente mayor de $\mathrm{RN}$ pequeños para edad gestacional (PEG) entre los RN de madres fumadoras diarias $(17,9 \%$ versus $9,6 \%)$; las características de los RN se presentan en la Tabla 2.

Cuando se contrastaron los pesos de los RN de madres que fumaron o no durante el embarazo y que estuvieron o no expuestas a humo de tabaco intradomiciliario, se observó que el peso promedio de los RN de madres que fumaron durante el embarazo y que estuvieron expuestas (3.329 g, IC95\% 3.230-3.428) y no expuestas (3.366 g, IC95\% 3.134-3.590), no fue significativamente diferente $(F=0,109, p=0,74)$. Por otra parte, el peso promedio de los RN de madres que no fumaron en el embarazo y que estuvieron expuestas (3.362 g, IC95\% 3.290$3.435)$ y no expuestas a tabaco intradomiciliario (3.396 g, IC95\% 3.301-3.490), tampoco fue significativamente diferente $(\mathrm{F}=0,31, \mathrm{p}=0,58)$.

En las madres que estuvieron expuestas a humo de tabaco intradomiciliario durante su embarazo, tampoco hubo diferencias significativas en el peso de los RN de acuerdo a si antes del embarazo eran fumadoras diarias (3.313 IC95\% 3.235-3.391), ocasionales (3.317 IC95\% 3.1553.480 ), o no fumaban (3.427 IC95\% 3.3283.526), (3.313 IC95\% 3.235-3.391), $F=1,6$; $\mathrm{p}=0,21$

De las madres que eran fumadoras diarias antes del embarazo (61,3\%), el 93,3\% fumaba entre 1-10 cigarrillos/día, el 4,8\% entre 11 y 20 cigarrillos/día y el 1,8\% más de 20 cigarrillos/ día. En comparación, las madres que dijeron fumar ocasionalmente, sólo el 26,3\% fumaba entre 1 y 10 cigarrillos, y ninguna informó fumar más de 10 cigarrillos, cuando fumaba.

No hubo diferencia significativa en el porcentaje de parto por cesárea entre las madres que fumaron $(12,8 \%)$ y las que no fumaron durante el embarazo (13,5\%), ni hubo asociación significativa entre la paridad previa (nulípara o multíparas) y el hecho de fumar o no durante todo el embarazo $(p=0,24)$. Tampoco se encontraron diferencias significativas entre las madres que fumaron o no durante el embarazo y la proporción de comorbilidades. Respectivamente, para las que fumaron versus las que no fumaron, los porcentajes fueron los siguientes: anemia $(13,5 \%$ vs $12,2 \%, \mathrm{p}=0,73)$; colestasia $(0,9 \%$ vs $2,4 \%, \mathrm{p}=0,45)$; ITU $(6,3 \%$ vs $10,8 \%$, $\mathrm{p}=0,19)$; toxemia $(11,7 \%$ vs $12,2 \%, \mathrm{p}=1,0)$; amenaza de aborto $(0,9 \%$ vs $1,0 \%, \mathrm{p}=1,0)$; amenaza de parto prematuro $(0,9 \%$ vs $1,0 \%$, $\mathrm{p}=1,0)$; diabetes gestacional $(0,0 \%$ vs $2,4 \%$, $\mathrm{p}=0,10)$; retardo crecimiento intrauterino $(0,9 \%$ vs $0,0 \%, \mathrm{p}=0,28)$.

\section{Discusión}

Este trabajo descriptivo demuestra que la prevalencia total de tabaquismo durante el embarazo en esta población de madres de bajo nivel socioeconómico fue de $28 \%$, tan alta como la informada recientemente en países desarrolla$\operatorname{dos}^{5-7}$ y mucho más alta que el $4,7 \%$ descrito en 
México ${ }^{8}$. En nuestra muestra, la prevalencia de tabaquismo diario previo al embarazo fue $61,3 \%$ siendo esa cifra mayor que la recientemente informada por CONACE que indica una prevalencia de $55 \%$ en el año 2004 en mujeres chilenas de 19-25 años (rango de edad de mayor prevalencia $)^{2}$. En Estados Unidos, las variaciones de la prevalencia del tabaquismo en embarazo oscilan entre $15,8 \%$ y $38,5 \%$.

En nuestro estudio se observó que el $42,6 \%$ de las fumadoras siguió fumando durante el primer trimestre. En un estudio multicéntrico en Europa, Torrent et $\mathrm{al}^{10}$ encontraron que en Barcelona, un $31,9 \%$ de las madres seguía fumando durante el primer trimestre de embarazo en comparación con un 20,4\% en Menorca y un $18 \%$ en Ashford, UK. Además, Torrent et $\mathrm{al}^{10}$ observaron que el $15,1 \%$ de las madres estudiadas volvió a fumar en los primeros días del puerperio, semejante al $12,6 \%$ encontrado en el presente estudio. En nuestro estudio, un 50,3\% de las madres fumadoras diarias y sólo un $9,6 \%$ de las madres fumadoras ocasionales siguieron fumando durante el primer trimestre del embarazo. Esa diferencia es compatible con la evidencia de que cuanto mayor es la intensidad del consumo de tabaco, mayor es el riesgo de seguir fumando durante el embarazo ${ }^{11}$. También es significativo, en términos de reincidencia del hábito, el hecho de que un alto porcentaje de las madres que dijeron que habían suspendido el consumo de tabaco cuando supieron que estaban embarazada haya continuado fumando posteriormente durante el embarazo.

Es lamentable que un alto porcentaje de las embarazadas (fumadoras y no fumadoras) estuvieran expuestas al humo de tabaco en sus hogares $(66 \%)$, lo cual también refleja la ignorancia existente en las personas que rodean a las mujeres embarazadas, en especial con respecto al efecto negativo en la salud humana causado por la exposición pasiva al humo del tabaco, el cual es sin duda uno de los más frecuentes y dañinos contaminantes intradomiciliarios.

Los efectos del consumo de tabaco durante el embarazo y su impacto en la salud del RN son abundantes y entre ellos se destacan el bajo peso al nacer, retardo de crecimiento intrauterino, el aumento de la tasa de aborto espontáneo y partos prematuros, el aumento de la morbilidad perinatal, síndrome de muerte súbita en lactante, disminución de la función pulmonar el primer año de vida, mayor riesgo de desarrollar asma en la niñez y mayor morbimortalidad por enfermedades respiratorias en la infancia ${ }^{12-18}$. Aunque en nuestro estudio no hubo diferencias significativas en los parámetros antropométricos de los RN de madres que fumaron o no durante el embarazo, el grupo de RN cuyas madres fumaron en el embarazo tuvo en promedio $100 \mathrm{~g}$ menos de peso al nacer que los niños del grupo no expuesto.

Está demostrado que la nicotina es un vasoconstrictor placentario y que reduce el flujo sanguíneo intravelloso ocasionando una disminución del aporte de nutrientes y oxígeno para el feto. Además la placenta de la madre fumadora es más pequeña, tiene mayor incidencia de infartos y demuestran con mayor frecuencia signos de perfusión inadecuada, lo cual revelaría el profundo efecto vascular de los derivados del tabaco ${ }^{19,20}$. Nuestro estudio, al demostrar que los recién nacidos de madres que eran fumadoras diarias antes del embarazo tienen un menor peso de nacimiento y una mayor frecuencia de retardo de crecimiento intrauterino, independientemente de si dejaron o no de fumar en el embarazo, sugiere un efecto muy precoz y persistente del tabaco sobre las estructuras vasculares del complejo útero-placenta-feto. De esta manera sería razonable inferir que en las mujeres fumadores en edad fértil, posiblemente los mecanismos responsables del efecto del tabaquismo materno sobre los RN (y también posteriormente en la niñez) estén establecidos antes del embarazo o en etapas muy iniciales de la gestación. Esto podría explicar, al menos en parte, el hallazgo de este estudio en el sentido que las madres que eran fumadoras diarias antes del embarazo dieron a luz recién nacidos con un peso promedio significativamente menor que el de los niños de madres que eran fumadoras ocasionales y de aquellas no fumadoras. En nuestro estudio el hecho que las madres suspendieran o no el tabaquismo al saber del embarazo no estableció diferencias en términos de peso de $\mathrm{RN}$, patología asociada al embarazo, ni en el tipo de parto.

La elevada prevalencia de tabaquismo durante el embarazo en madres adolescentes encontrada en nuestro estudio, sumada a la información reciente del CDC (Center for Disease Control and Prevention, USA) de una menor eficacia de las campañas anti-tabaco en adolescentes embarazadas $^{7}$, alerta sobre la necesidad de desarrollar nuevas intervenciones educacionales y preventivas que empleen medios comunicacionales modernos y preferidos por los niños (ej: Internet) dirigidas específicamente a niños y adolescentes, además de restringir seriamente la publicidad y venta de cigarrillos con el objetivo de contrarrestar el potente estímulo mediático de la 
industria tabacalera para iniciar tempranamente y continuar el consumo de tabaco.

En conclusión, este estudio descriptivo demuestra que el $28 \%$ de las mujeres embarazadas de bajo nivel socioeconómico fuma durante la gestación. El porcentaje de tabaquismo durante el embarazo en la población estudiada es tan alto como los descritos en mujeres embarazadas de países desarrollados. Destacan la mayor prevalencia de tabaquismo durante el embarazo en madres adolescentes y el alto porcentaje de mujeres embarazadas, fumadoras y no fumadoras, expuestas a humo de tabaco en sus hogares. Los resultados de este estudio también se suman a la amplia evidencia existente acerca del efecto deletéreo del consumo de tabaco materno en la salud de las madres y de los recién nacidos.

\section{Bibliografía}

1.- Banco Mundial-OPS/OMS. La Epidemia de Tabaquismo. Los Gobiernos y los Aspectos Económicos del Control del Tabaco. Washington DC. Publicación Científica $\mathrm{N}^{\circ} 577,2000$.

2.- Ministerio del Interior de Chile, Consejo Nacional para el Control de Estupefacientes, CONACE, Estadísticas, Consumo de cigarrillos en Chile, Mayo 2005. www.conacedrogas.cl

3.- GOLDENBERG R L, KLERMAN L V, WINDSOR R A, WHITESIDE H P Jr. Smoking in pregnancy: Final thoughts. Tob Control 2000; 9: Suppl 3: III85-6.

4.- JUEZ G, LUCERO E, VENTURA-JUNCÁ P, TAPIA J L, GONZÁLEZ H, WINTER A. Intrauterine growth in Chilean middle class newborn infants. Rev Chil Pediatr 1989; 60: 198-202.

5.- CHATENOUD L, CHIAFFARINO F, PARAZZINI F, BENZI G, LA VECCHIA C. Prevalence of smoking among pregnant women is lower in Italy than England. BMJ 1999; 318: 1012.

6.- OWEN L, MCNEILL A, CALLUM C. Trends in smoking during pregnancy in England, 1992-7: quota sampling surveys. BMJ 1998; 317: 728-30.

7.- Center for disease control and prevention, MMWR. Smoking during prernancy-United States, 1990-2002. Oct 8, 2004; 53 (39): 911-5

8.- SÁNCHEZ-ZAMORANO M L, TÉLLEZ-ROJO M M, HERNÁNDEZ-ÁVILA M. Effect of smoking during pregnancy on anthropometric characteristics at birth. Salud Púb Mex 2004; 46: 529-33.

9.- LIPSCOMB L E, JOHNSON C H, MORROW B. PRAMS 1998 Surveillance Report. Atlanta: Division of Reproductive Health, National Center for Chronic Disease Prevention and Health Promotion, Centers for Disease Control and Prevention. www.cdc.gov/ prams/Reports.htm

10.- TORRENT M, SUNYER J, CULLINAN P, BASAGANA X, HARRIS J, GARCÍA O, et al. Smoking cessation and associated factors during pregnancy. Gac Sanit 2004; 18: 184-9.

11.- DOZ MOURA J, GASULLA PASCUAL G, CARCELES JURADO S, GUERRERO BARTOLIN I, TINTO PADRO A, PRAT ASENCIO C, et al. Evolution of tobacco habits during pregnancy and postpartum period. Aten Primaria 2004; 34: 465-71.

12.- MELVIN C L, ADAMS E K, MILLER V. Cost of smoking during pregnancy: development of the maternal and child health smoking attributable mortality, morbity and economic costs (MCHSAMMEC software). Tob Control 2000; 9 Suppl 3: III12-5.

13.- CASTLES A, ADAMS E K, MELVIN C L, KELSCH C, BOULTON M L. Effects of smoking during pregnancy. Five meta-analyses. Am J Prev Med 1999; 16: 208-15.

14.- COOKE R W J. Smoking, intra-uterine growth retardation and sudden infant death syndrome. Int $\mathrm{J}$ Epidem 1998; 27: 238-41.

15.- DEZATEUX C, STOCKS J, DUNDAS I, FLETCHER M E. Impaired Airway Function and Wheezing in Infancy: The influence of maternal smoking and a genetic predisposition to asthma. Am J Respir Crit Care Med 1999; 159: 403-10.

16.- MORGAN W J. Maternal smoking and infant lung function: further evidence for an in utero effect. Am J Respir Crit Care Med 1998; 158: 689-90.

17.- GILLILAND F D, LI Y F, PETERS J M. Effects of maternal smoking during pregnancy and environmental tabacco smoke on asthma and wheezing in children. Am J Resp Crit Care Med 2001; 163: 429-36.

18.- GERGEN P J, FOWLER J A, MAURER K R, DAVIS W W, OVERPECK M D. The burden of environmental tobacco smoke exposure on the respiratory health of children 2 month through 5 years of age in the United States: Third National Health and Nutrition Examination Survey, 1988 to 1994. Pediatrics 1998; 101: 8-14

19.- WALSH R A. Effects of maternal smoking on adverse pregnancy out-comes: examination of the criteria of causation. Hum Biol 1994; 66: 1059-92.

20.- ASMUSSEN I. Ultrastructure of the human placenta at term. Observations on placentas from newborn children of smoking and non-smoking mothers. Acta Obstet Gynecol Scand 1977; 56: 119-26.

Correspondencia a:

Dr. Javier Mallol

Casilla 23 Correo 9. Santiago, Chile

Fax: 5623874662

E-mail: jmallol@usach.cl 